\title{
Alterações motoras após cirurgia refrativa no paciente estrábico
}

\author{
Motor disturbances after refractive surgery in patients with strabismus
}

\author{
Rosana Nogueira Pires da Cunha ${ }^{1}$ \\ Inez Eloísa Isoldi ${ }^{2}$ \\ MarceloCunha $^{3}$
}

\section{RESUMO}

Objetivo: Estudar a evolução clínica de pacientes com estrabismo e que se submeteram à cirurgia refrativa. Métodos: Foram examinados 15 pacientes de outubro de 2000 a setembro de 2001 com história de estrabismo e cirurgia fotorrefrativa, incluindo avaliação sensório-motora. O tempo de seguimento foi no mínimo de 6 meses da correção a laser. Os pacientes que já haviam sido submetidos à correção a laser constituíram o primeiro grupo, e os que foram examinados antes da correção a laser, formaram o segundo grupo. Resultados: Todos os pacientes do primeiro grupo necessitaram de cirurgia corretora de estrabismo para aliviar os sintomas de visão dupla ou corrigirem o aumento do desvio ocular. A cirurgia fotorrefrativa foi realizada nesses casos em pacientes com estrabismo pré-existente e sem avaliação prévia de suas condições sensoriais.A maioria dos pacientes do segundo grupo apresentou piora da visão binocular após o procedimento a laser. Metade dos pacientes desse grupo precisou usar óculos para detalhes de perto para aliviar sintomas de astenopia. Aqueles que possuíam esotropia acomodativa foram os que apresentaram menos ou nenhuma queixa após o procedimento, e a evolução foi estável ao longo dos meses de pós-operatório. Conclusões: Os cirurgiões refrativos devem selecionar seus pacientes, evitando subestimar alterações que possam comprometer a capacidade de fusão após o laser. Recomendamos a avaliação sensório-motora detalhada, antes do laser, para todos os candidatos à cirurgia refrativa.

Descritores: Cirurgia a laser/efeitos adversos; Estrabismo/cirurgia; Estrabismo/complicações

Trabalho realizado na Clínica de Olhos Dr. Moacir Cunha - São Paulo (SP).

${ }^{1}$ Oftalmologista da Clínica de Olhos Dr. Moacir Cunha e do Departamento de Oftalmologia da Universidade Federal de São Paulo - UNIFESP, com pós-graduação nível doutorado na mesma instituição.

Ortoptista da Clínica de Olhos Dr. Moacir Cunha.

${ }^{3}$ Oftalmologista da Clínica de Olhos Dr. Moacir Cunha e do Departamento de Oftalmologia da Universidade Federal de São Paulo - UNIFESP, com pós-graduação nível doutorado na mesma instituição.

Endereço para correspondência: Rosana Pires da Cunha - Alameda Gabriel Monteiro da Silva, 1000, São Paulo (SP) CEP 01442-000

E-mail: rocunha@netpoint.com.br

Recebido para publicação em 20.11.2002

Versão revisada recebida em 06.05.2003

Aprovação em 31.10.2003

Nota Editorial: Pela análise deste trabalho e por sua anuência na divulgação desta nota, agradecemos ao Dr. Renato Luiz Nahoum Curi.

\begin{abstract}
INTRODUÇÃO
A cirurgia refrativa bem sucedida traz mudanças muito positivas na qualidade de vida da maioria dos pacientes, e o sucesso traz popularidade: a projeção para o ano de 2002, de cirurgias refrativas a laser no mundo, é de 4 milhões ${ }^{(1)}$. A população ouve falar em uma sociedade livre de óculos e de poder mudar totalmente sua vida, contagiando os próprios oftalmologistas.

Apesar de vários estudos mostrarem que a cirurgia refrativa é geralmente segura ${ }^{(2-3)}$, complicações podem ocorrer, como a presença de halos, "glare", hipo ou hipercorreções e infecções de córnea ${ }^{(4)}$.

Alguns casos de estrabismo, com ou sem diplopia, têm sido relatados ${ }^{(5-9)}$. Os motivos podem ser diversos, como perda da supressão, descompensação de estrabismo pré-existente e monovisão planejada em pacientes com fusão instável ${ }^{(10)}$. Apesar de raros, esses estrabismos interferem no resultado final e sua incidência tende a aumentar proporcionalmente ao número de casos operados. A partir da constatação dessas complicações em nosso meio, após
\end{abstract}


exame de um grupo de pacientes que possuíam queixas relacionadas a distúrbios da motilidade ocular, e que foram desencadeadas ou agravadas após a cirurgia refrativa, analisamos a evolução clínica de um grupo de pacientes com história pregressa de estrabismo ou ambliopia, antes e após o laser.

\section{MÉTODOS}

Foram examinados, de outubro de 2000 a setembro de 2001, 15 pacientes com história de estrabismo na infância ou adolescência, com idade média de $36,33 \pm 11,32$ (variando de 20 a 65 anos). Dez eram do sexo feminino e cinco eram do sexo masculino.

Os primeiros cinco pacientes que examinamos já haviam sido submetidos à cirurgia refrativa e apresentavam queixas de visão dupla, astenopia ou aumento do desvio ocular pré-existente. Este grupo, chamado de grupo 1, foi o que justificou este estudo, pois a partir destes casos, começamos a fazer avaliação ortóptica em todos os pacientes que desejavam fazer a correção a laser e apresentavam história pregressa de estrabismo e/ou ambliopia. Portanto, os outros dez pacientes que foram examinados antes e após a correção a laser, formaram o grupo 2 .

A avaliação dos pacientes de ambos grupos consistiu em exame ocular completo (AV com e sem correção, refratometria dinâmica e estática, biomicroscopia, tonometria e fundo de olho), incluindo avaliação da motilidade ocular. O exame sensorial consistiu em avaliação da visão binocular com o teste de Titmus (estereopsia) e com os vidros estriados de Bagolini (pesquisa de fusão ou supressão). Este exame só pôde ser realizado nos pacientes do grupo 1 após a cirurgia refrativa, e nos pacientes do grupo 2 , antes e após o procedimento.

O tempo de seguimento foi no mínimo de 12 meses após a correção a laser e em todos os pacientes utilizou-se a técnica de LASIK.

O quadro 1 relaciona os dados dos pacientes do Grupo 1. Estes pacientes foram encaminhados de outros serviços por apresentarem queixas de visão dupla, astenopia e/ou aumento de desvio pré-existente. Todos relatavam que esses sintomas pioraram ou começaram após a correção a laser. Na primeira consulta realizada em nosso serviço foi prescrita a refração estática para todos que apresentavam desvio convergente e a avaliação motora e sensorial da motilidade ocular foi realizada sob uso desta refração, com o objetivo de eliminar a influência de qualquer fator acomodativo.

Os pacientes do Grupo 2 foram examinados antes de serem submetidos à cirurgia refrativa, incluindo avaliação sensóriomotora com a refração estática, pelo mesmo motivo citado para os pacientes do Grupo 1 (Quadro 2). Os pacientes que não possuíam visão binocular central foram submetidos à avaliação de suas condições sensório-motoras com o uso de lentes de contato hipocorrigidas. $\mathrm{O}$ objetivo deste teste era avaliar a relação da acomodação com seu estrabismo, caso a cirurgia refrativa resultasse em hipocorreção. Após um mês da cirurgia a laser, os pacientes eram submetidos a novo exame ortóptico, mesmo sem queixas (Quadro 3).

\section{RESULTADOS}

O quadro 1 demonstra os dados dos pacientes do Grupo 1 . A maioria (4/5 pacientes) era hipermétrope antes do laser. Os pacientes numerados de 1 a 4 apresentavam desvio convergente e ficaram com discreto grau residual de hipermetropia ou astigmatismo hipermetrópico após a cirurgia refrativa, exceto um (paciente 3) que ficou hipercorrigido (míope) em um olho e passou a usar esse olho para perto e o outro para longe. Todos os pacientes desse grupo apresentavam acuidade visual igual ou maior que 0,9 com correção. Dos quatro pacientes que apresentavam desvio convergente, um deles (paciente 1), que era o único míope do Grupo 1, apresentou um quadro de descompensação de desvio latente (esoforia), ficando em desvio manifesto (esotropia) logo após o procedimento. Outros dois (pacientes 2 e 4) apresentavam microesotropia associada a fator acomodativo e incomitância alfabética ou componente vertical, fatores que podem ter contribuído para a descompensação do desvio pré-existente. Somente um paciente desse grupo apresentava desvio divergente, que antes era intermitente e passou a se manifestar de forma constante, segundo a paciente, logo após a correção a laser ter sido realizada em um dos olhos.

$\mathrm{O}$ quadro 2 demonstra os dados dos pacientes do Grupo 2 antes da cirurgia fotorrefrativa. A maioria ( $9 / 10$ pacientes) era hipermétrope e apresentava desvio convergente, exceto aquele que era míope (paciente 6) e apresentou espasmo de acomodação após a correção. Todos os pacientes do Grupo 2 apresentavam visão binocular central ou periférica.

O quadro 3 descreve os dados dos pacientes do Grupo 2 após o laser. Oito (8/10) deles apresentaram bons resultados (refração dinâmica igual ou menor que 1,00 dioptrias esféricas em equivalentes esféricos), com acuidade visual igual ou maior que 0,9 sem correção. Um (1/10) deles (paciente 2) ficou hipocorrigido em um olho, mas mesmo com acuidade visual mais baixa não apresentou piora de seu estrabismo até o retratamento. Outro (1/10) (paciente 4) possuía ambliopia e seu estrabismo também não piorou. Cinco (5/10) pacientes apresentavam alguma queixa relativa à visão ou a desvio ocular. Destes, um (paciente 1) notou visão dupla no pós-operatório imediato e foi constatado hipocorreção (hipermetropia residual), sendo tratada com óculos para serem usados em atividades com detalhes para perto, os quais foram também prescritos para cinco $(5 / 10)$ pacientes a fim de aliviar a astenopia, diplopia ou sensação de desvio ocular.

\section{DISCUSS ÃO}

Existem poucas publicações a respeito das alterações na motilidade ocular após cirurgia refrativa, possivelmente por não serem relatadas quando ocorrem ou mesmo por serem totalmente negligenciadas pelos cirurgiões refrativos.

Entretanto, comentários ou relatos de casos de pacientes com visão dupla têm aumentado em freqüência. $\mathrm{O}$ primeiro relato foi em $1987^{(5)}$, sobre um paciente que havia feito corre- 


\begin{tabular}{|c|c|c|c|c|c|c|c|c|c|}
\hline \multirow[t]{2}{*}{ Pac } & \multirow{2}{*}{$\begin{array}{l}\text { Idade/ } \\
\text { sexo }\end{array}$} & \multirow{2}{*}{$\begin{array}{l}\text { História } \\
\text { pregressa }\end{array}$} & \multirow{2}{*}{$\begin{array}{l}\text { Avaliação } \\
\text { sensório- } \\
\text { motora antes } \\
\text { do laser }\end{array}$} & \multirow{2}{*}{$\begin{array}{l}\text { Após o laser } \\
\text { queixa/ } \\
\text { duração }\end{array}$} & \multicolumn{2}{|c|}{ Refração estática (AV) } & \multirow{2}{*}{$\begin{array}{l}\text { Avaliação } \\
\text { sensório- } \\
\text { motora } \\
\text { pós-laser }\end{array}$} & \multirow[t]{2}{*}{ Diagnóstico } & \multirow{2}{*}{$\begin{array}{l}\text { Conduta/ } \\
\text { evolução }\end{array}$} \\
\hline & & & & & Pré-Laser & Pós-Laser & & & \\
\hline 1 & $\begin{array}{l}28 \\
M\end{array}$ & $\begin{array}{l}\text { Olho desviava } \\
\text { na adolescên- } \\
\text { cia - às vezes } \\
\text { visão dupla } \\
\text { - não fez } \\
\text { tratamento }\end{array}$ & $\begin{array}{l}E(T) 12 \text { +foria } \\
\text { Baixa } \\
\text { amplitude } \\
\text { fusional VB } \\
\text { periférica }\end{array}$ & $\begin{array}{l}\text { Diplopia há } 3 \\
\text { meses, desde } \\
\text { o laser }\end{array}$ & $\begin{array}{l}\text { OD }-0,75 \text { de } \\
\text { OE }-1,00 \text { de }\end{array}$ & $\begin{array}{l}\text { OD }+0,50 \text { de } \\
-0,50 \mathrm{dc} \\
\times 180(0,9) \\
\text { OE }+1,00 \mathrm{de} \\
(1,0)\end{array}$ & $\begin{array}{l}\text { ET } 25 \\
\text { ET' } 16 \\
\text { Fusão na } \\
\text { medida } \\
\text { objetiva }\end{array}$ & $\begin{array}{l}\text { ET não } \\
\text { acomodativa }\end{array}$ & $\begin{array}{l}\text { Correção da } \\
\text { diplopia com } \\
\text { prismas por } \\
1 \text { mês } \\
\text { Cirurgia/ } \\
\text { Ortotropia }\end{array}$ \\
\hline 2 & $\begin{array}{l}29 \\
F\end{array}$ & $\begin{array}{l}\text { Estrabismo } \\
\text { na infância } \\
\text { - óculos } \\
\text { oclusão e } \\
\text { exercícios }\end{array}$ & $\begin{array}{l}\text { sc } \mathrm{E}(\mathrm{T}) 15 \\
\text { cc micro ET } \\
\text { VB periférica }\end{array}$ & $\begin{array}{l}\text { Astenopia e } \\
\text { aumento do } \\
\text { desvio há um } \\
\text { ano, após } 6 \\
\text { meses do laser }\end{array}$ & $\begin{array}{l}\mathrm{OD}+1,25 \mathrm{de} \\
-0,25 \mathrm{dc} \times 180 \\
\mathrm{OE}+3,00 \mathrm{de} \\
-1,25 \mathrm{dc} \times 5\end{array}$ & $\begin{array}{l}\text { OD }+1,25 \text { de } \\
(1,0) \\
\text { OE }+1,25 \text { de } \\
(1,0)\end{array}$ & $\begin{array}{l}\mathrm{E}(\mathrm{T}) 16 \\
\mathrm{E}(\mathrm{T}))^{\prime} 25 \\
\text { Micro } \mathrm{ET} 4 \\
\text { VB periférica }\end{array}$ & $\begin{array}{l}\text { ET } \\
\text { parcialmente } \\
\text { acomodativa } \\
\text { e incomitância } \\
\text { em V }\end{array}$ & $\begin{array}{l}\text { Cirurgia e } \\
\text { prescrição } \\
\text { para leitura / } \\
\text { Micro ET } \\
\text { com FA }\end{array}$ \\
\hline 3 & $\begin{array}{l}55 \\
F\end{array}$ & $\begin{array}{l}\text { Notou desvio } \\
\text { quando já era } \\
\text { adulta, sem } \\
\text { alterações } \\
\text { neurológicas. } \\
\text { Fotos ante- } \\
\text { riores - já } \\
\text { possuía ET }\end{array}$ & $\begin{array}{l}\text { sc ET } 20 \\
\text { cC E } 10 \\
\text { VB periférica }\end{array}$ & $\begin{array}{l}\text { Aumento do } \\
\text { desvio e diplo- } \\
\text { pia há } 1 \text { ano, } \\
\text { piorou logo } \\
\text { após o laser. } \\
\text { Fecha OD para } \\
\text { atividades à } \\
\text { distância }\end{array}$ & $\begin{array}{l}\mathrm{OD}+3,00 \mathrm{de} \\
\mathrm{OE}+2,25 \mathrm{de}\end{array}$ & $\begin{array}{l}\text { OD }+0,50 \mathrm{de} \\
-0,50 \mathrm{dc} \\
\times 155(1,0) \\
\text { OE }-2,00 \mathrm{de} \\
-0,75 \mathrm{dc} \\
\times 10(0,9)\end{array}$ & $\begin{array}{l}\text { ET } 20 \\
\text { ET' } 7 \\
\text { Fusão na } \\
\text { medida } \\
\text { objetiva }\end{array}$ & $\begin{array}{l}\text { ET com } \\
\text { incomitância } \\
\text { em A }\end{array}$ & $\begin{array}{l}\text { Adaptação } \\
\text { de LC no OE/ } \\
\text { Cirurgia/ } \\
\text { Ortotropia }\end{array}$ \\
\hline 4 & $\begin{array}{l}39 \\
M\end{array}$ & $\begin{array}{l}\text { Cirurgia de } \\
\text { estrabismo } \\
\text { na infância }\end{array}$ & $\begin{array}{l}\text { sc ET } 30 \\
\text { cc ET } 10 \\
\text { Ausência } \\
\text { de VB }\end{array}$ & $\begin{array}{l}\text { Aumento do } \\
\text { desvio há } \\
2 \text { anos e } \\
\text { astenopia } \\
\text { desde o laser }\end{array}$ & $\begin{array}{l}\text { OD }+3,00 \text { de } \\
-2,00 \text { dc } \times 155 \\
\text { OE }+3,75 \text { de } \\
-2,50 \text { dc } \times 25\end{array}$ & $\begin{array}{l}\text { OD }+0,75 \text { de } \\
(1,0) \\
\text { OE }+1,00 \text { de } \\
-0,50 \text { dc } \\
x 90(1,0)\end{array}$ & $\begin{array}{l}\text { ET } 20 \\
\text { ET' } 25\end{array}$ & $\begin{array}{l}\text { Ausência } \\
\text { de VB }\end{array}$ & $\begin{array}{l}\text { Micro ET com } \\
\text { FA e compo- } \\
\text { nente vertical } \\
\text { Cirurgia/ } \\
\text { Micro ET }\end{array}$ \\
\hline 5 & 65 & $\begin{array}{l}\text { Tratamento } \\
\text { ortóptico na } \\
\text { infância }\end{array}$ & $\begin{array}{l}\text { cc e sc } \\
X(T) 20 \text { VB } \\
\text { perturbada }\end{array}$ & $\begin{array}{l}\text { Aumento do } \\
\text { desvio e } \\
\text { astenopia } \\
1 \text { ano após } \\
\text { o laser }\end{array}$ & $\begin{array}{l}\mathrm{OD}+3,00 \mathrm{de} \\
\mathrm{OE}+4,00 \mathrm{de}\end{array}$ & $\begin{array}{l}\text { OD plano } \\
(1,0) \\
\text { OE }-0,50 \text { de } \\
-0,25 \mathrm{dc} \\
\times 15(1,0)\end{array}$ & $\begin{array}{l}\text { XT } 20 \\
\text { HTE } 6 \\
\text { XT' } 20 \\
\text { HTE } 6\end{array}$ & $\begin{array}{l}\text { Ausência } \\
\text { de VB }\end{array}$ & $\begin{array}{l}\text { XT } \\
\text { Cirurgia / } \\
\text { Micro XT }\end{array}$ \\
\hline
\end{tabular}

ção de esotropia há muitos anos e após ceratotomia radial para correção de alta miopia no primeiro olho, apresentou recidiva do desvio convergente e diplopia. Após alguns anos, foi relatado um caso semelhante ${ }^{(6)}$, também após ceratotomia radial.

O primeiro caso descrito de descompensação de estrabismo após cirurgia fotorrefrativa foi relacionado à monovisão induzida em uma paciente míope e présbita ${ }^{(7)}$. Apesar de negar tratamento anterior de estrabismo, a paciente desenvolveu diplopia decorrente de desvio vertical e torsional associado a desvio divergente de grande ângulo. Esse quadro foi caracterizado como paresia do músculo oblíquo superior associado a exotropia e a visão dupla só foi compensada com cirurgia corretora de estrabismo.

Em 1999 foi descrito um caso de descompensação de paresia de oblíquo superior após monovisão induzida por LASIK, no qual o desvio pôde ser compensado com prismas ${ }^{(8)}$. Outro caso de monovisão induzida resultando em diplopia foi descrito $^{(9)}$, ocorrendo após um mês do LASIK para correção de miopia monocular.
Os pacientes do grupo 1, descritos neste estudo (Quadro 1), são exemplos de desvios horizontais secundários a descompensação e provável perda da binocularidade após cirurgia fotorrefrativa, semelhante aos casos descritos após ceratotomia radial ${ }^{(5-6)}$. Baseando-se na análise retrospectiva das respectivas fichas médicas, notamos que todos os pacientes desse grupo tinham um ponto em comum: seu estado sensório-motor não foi avaliado e conseqüentemente não foi valorizado antes da cirurgia refrativa. Um dos pacientes descritos (paciente 1, Quadro 1) apresentava esotropia comitante adquirida quando jovem, associada à miopia (Figura 1). Esse paciente relatava que esporadicamente apresentava visão dupla antes da cirurgia refrativa, perdendo totalmente a capacidade de fusão após o laser, com diplopia constante. O motivo da perda da supressão e rompimento da fusão justamente após o laser nesse caso não é conhecido, mas se o paciente tivesse sido submetido à avaliação sensório-motora, provavelmente seria demonstrada baixa capacidade fusional.

Os dois pacientes do Grupo 1 que possuíam esotropia parcial- 


\begin{tabular}{|c|c|c|c|c|c|c|c|c|}
\hline \multicolumn{9}{|c|}{ Quadro 2. Descrição dos casos do grupo 2 - Exame realizado antes do laser em pacientes que desejavam a cirurgia } \\
\hline Pac & Idade & Sexo & HP & $\begin{array}{l}\text { Refração em } \\
\text { uso (AV) }\end{array}$ & $\begin{array}{l}\text { Refração } \\
\text { estática }\end{array}$ & MOE-PPO & $\begin{array}{l}\text { Avaliação } \\
\text { sensorial }\end{array}$ & Diagnóstico \\
\hline 1 & 27 & $\mathrm{~F}$ & $\begin{array}{l}\text { Estrabismo na } \\
\text { infância - óculos } \\
\text { e exercícios } \\
\text { ortópticos }\end{array}$ & $\begin{array}{l}\mathrm{OD}+2,25 \mathrm{de} \\
-2,00 \mathrm{dc} \times 175(1,0) \\
\mathrm{OE}+2,50 \mathrm{de} \\
-2,25 \mathrm{dc} \times 80(1,0)\end{array}$ & $\begin{array}{l}\text { OD }+3,25 \text { de } \\
-1,75 d c \times 180 \\
\text { OE }+3,50 \text { de } \\
-2,00 \text { dc } \times 5\end{array}$ & $\begin{array}{l}\text { sc ET } 10 ; \\
\text { ET' } 5 \\
\text { cc ortotropia; } \\
\text { ET' } 4\end{array}$ & VB periférica & MicroET com FA \\
\hline 2 & 29 & M & $\begin{array}{l}\text { Estrabismo na } \\
\text { infância - óculos, } \\
\text { cirurgia }\end{array}$ & $\begin{array}{l}\text { OD }+5,00 \text { de } \\
-4,50 \text { dc } \times 15(0,5) \\
\text { OE }+4,50 \text { de } \\
-3,25 \text { dc } \times 165(0,8)\end{array}$ & $\begin{array}{l}\text { OD }+6,00 \text { de } \\
-5,00 d c \times 15 \\
O E+4,50 \text { de } \\
-3,50 d c \times 165\end{array}$ & $\begin{array}{l}\text { Sc XT 5; } \\
\text { ET' } 5 \\
\text { cc XT 5; } \\
\text { ET' } 4\end{array}$ & VB periférica & $\begin{array}{l}\text { Síndrome da } \\
\text { monofixação } \\
\text { com FA; ambliopia }\end{array}$ \\
\hline 3 & 32 & $\mathrm{~F}$ & $\begin{array}{l}\text { Estrabismo na } \\
\text { infância - óculos }\end{array}$ & $\begin{array}{l}\text { OD }+5,50 \mathrm{de} \\
-1,50 \mathrm{dc} \times 40(1,0) \\
\mathrm{OE}+5,00 \mathrm{de} \\
-1,50 \mathrm{dc} \times 5(1,0)\end{array}$ & $\begin{array}{l}\text { OD }+6,25 \text { de } \\
-2,00 \mathrm{dc} \times 25 \\
\mathrm{OE}+6,25 \mathrm{de} \\
-2,00 \mathrm{dc} \times 180\end{array}$ & $\begin{array}{l}\text { sc } E(T)^{\prime}=10 \\
E(T)=7 \\
\text { cc } E(T)^{\prime}=4 \\
L \text { ortotropia }\end{array}$ & VB central & ET acomodativo \\
\hline 4 & 45 & M & $\begin{array}{l}\text { Óculos e oclusão } \\
\text { na infância }\end{array}$ & $\begin{array}{l}\text { OD }+4,00 \text { de } \\
-0,75 \text { dc } \times 20(0,9) \\
\text { OE }+3,00 \text { de }(1,0)\end{array}$ & $\begin{array}{l}\text { OD }+5,50 \text { de } \\
-1,00 \mathrm{dc} \times 170 \\
\text { OE }+4,25 \mathrm{de} \\
-1,00 \mathrm{dc} \times 180\end{array}$ & $\begin{array}{l}\text { sc ortotropia } \\
E(T)^{\prime} 4 \\
\text { cc ortotropia } \\
E(T)^{\prime} 4\end{array}$ & VB central & ET acomodativo \\
\hline 5 & 30 & $\mathrm{~F}$ & $\begin{array}{l}\text { Estrabismo na } \\
\text { infância - óculos } \\
\text { e oclusão }\end{array}$ & $\begin{array}{l}\text { OD }+3,50 \text { de }(0,9) \\
O E+4,00 \text { de }(1,0)\end{array}$ & $\begin{array}{l}\mathrm{OD}=+3,50 \mathrm{de} \\
\mathrm{OE}=+3,25 \mathrm{de}\end{array}$ & $\begin{array}{l}\text { sc ET } 15 \\
\text { ET' } 18 \\
\text { cc ET } 4 \\
\text { ET' } 7\end{array}$ & VB periférica & Micro ET com FA \\
\hline 6 & 43 & M & $\begin{array}{l}\text { Estrabismo na } \\
\text { infância }\end{array}$ & $\begin{array}{l}\text { OD }-2,50 \text { de } \\
-0,25 \mathrm{dc} \times 175(1,0) \\
\text { OE }-2,25 \mathrm{de} \\
-0,25 \mathrm{dc} \times 10(1,0)\end{array}$ & $\begin{array}{l}\text { OD }-1,75 \text { de } \\
-0,50 \text { dc } \times 10 \\
\text { OE }-2,00 \text { de }\end{array}$ & $\begin{array}{l}\mathrm{sc}=\mathrm{cc} \\
\text { ortotropia }\end{array}$ & VB central & $\begin{array}{l}\text { Espasmo de } \\
\text { acomodação }\end{array}$ \\
\hline 7 & 38 & $\mathrm{~F}$ & $\begin{array}{l}\text { Cirurgia de ET } \\
\text { na infância }\end{array}$ & $\begin{array}{l}\text { OD }+3,50 \text { de }(1,0) \\
O E+4,50 \text { de }(1,0)\end{array}$ & $\begin{array}{l}\mathrm{OD}+4,50 \mathrm{de} \\
\mathrm{OE}+5,50 \mathrm{de}\end{array}$ & $\begin{array}{l}\text { Sc ET } 12 \\
\text { HTE } 4 \\
\text { cC ET } 4 \\
\text { HTE } 4\end{array}$ & VB periférica & Micro ET com FA \\
\hline 8 & 34 & $\mathrm{~F}$ & ET infância & $\begin{array}{l}+5,00 \text { de } \\
-1,00 \text { dc } \times 180(1,0) \\
+5,25 \text { de } \\
-1,00 \text { dc } \times 180(1,0)\end{array}$ & $\begin{array}{l}+5,75 \text { de } \\
-0,75 \text { dc } \times 15 \\
+6,00 \text { de } \\
-1,00 \text { dc } \times 165\end{array}$ & $\begin{array}{l}\operatorname{sc} E(T) 20 \\
E(T)^{\prime} 15 \\
\text { cc E } 8 E^{\prime} 8\end{array}$ & VB central & ET acomodativo \\
\hline 9 & 31 & $\mathrm{~F}$ & ET infância & $\begin{array}{l}+1,00 \text { de } \\
-1,00 \mathrm{dc} \times 180(1,0) \\
+1,00 \mathrm{de} \\
-1,50 \mathrm{dc} \times 180(1,0)\end{array}$ & $\begin{array}{l}+3,00 \text { de } \\
-1,50 \text { dc } \times 5 \\
+3,25 \text { de } \\
-1,25 \text { dc } \times 180\end{array}$ & $\begin{array}{l}\text { sc } E(T) 12 \\
E(T)^{\prime} 12 \\
\text { cc } E(T) 12 \\
E(T)^{\prime} 8\end{array}$ & VB central & ET acomodativo \\
\hline 10 & 20 & $\mathrm{~F}$ & $\begin{array}{l}\text { Óculos e oclusão } \\
\text { na infância }\end{array}$ & $\begin{array}{l}+4,50 \text { de } \\
-2,00 \text { dc } \times 100(0,5) \\
+4,25 \text { de } \\
-2,00 \text { dc } \times 60\end{array}$ & $\begin{array}{l}+5,75 \text { de } \\
-2,00 \mathrm{dc} \times 100 \\
+5,00 \mathrm{de} \\
-2,00 \mathrm{dc} \times 60\end{array}$ & $\begin{array}{l}\text { Sc E } 12 \\
E(T)^{\prime} 12 \\
\text { cc E } 4 \\
E^{\prime} 4\end{array}$ & VB central & ET acomodativo \\
\hline
\end{tabular}

mente acomodativa (Quadro 1, pacientes 2 e 4) possuíam as mesmas queixas: astenopia e aumento do desvio progressivo após o laser. Após a correção cirúrgica do estrabismo eles apresentaram microesotropia, que poderia ser seu estado motor anterior. É interessante salientar que ambos pacientes aceitaram usar, por algum tempo, a refração estática que desenvolveram alguns meses após o laser, mas não houve modificação do desvio.

Um dos pacientes do Grupo 1 (paciente 3 - Quadro 1) apresentou diplopia após ficar com monovisão por hipercorreção não proposital em um dos olhos. Ao exame, além da esotropia, havia um fator importante de descompensação do des- vio, a incomitância alfabética em A, possivelmente uma característica prévia ao laser. Segundo concluem ${ }^{(7-8)}$ a perda da visão binocular de aparecimento após indução proposital de monovisão, deve-se a descompensação de um quadro que provavelmente já existia antes da correção a laser, e que era compensado graças à boa amplitude de vergência fusional (horizontal ou vertical). Com a indução proposital de monovisão, devido à disparidade de foco, é maior a dificuldade para a fusão das imagens, o que leva alguns pacientes a não mais conseguir compensar o desvio: de um estado de foria (desvio latente) ficam em estado de tropia, com visão dupla constante. 


\begin{tabular}{|c|c|c|c|c|c|c|}
\hline \multicolumn{7}{|c|}{ Quadro 3 - Descrição dos casos do grupo 2 - Exame realizado após o laser } \\
\hline Paciente & Refração dinâmica & Queixas & MOE-PPO & Avaliação sensorial & Conduta & Evolução \\
\hline 1 & AO plano $(1,0)$ & $\begin{array}{l}\text { Diplopia no } \\
\text { primeiro mês PO }\end{array}$ & $\begin{array}{l}\text { sc ET } 10 ; \\
\text { ET' } 12 \text { com RE } \\
(O D+2,25 \text { de } \\
-0,50 \text { dc } \times 120 \\
\text { OE }+2,50 \text { de) } \\
\text { cc E } 10 ; E^{\prime} 12\end{array}$ & VB central & $\begin{array}{l}\text { Prescrição de } \\
\text { óculos para perto } \\
\text { - diplopia cedeu }\end{array}$ & $\begin{array}{l}\text { Após um ano } \\
\text { cc ET 15; ET' } 4 \\
\text { VB periférica }\end{array}$ \\
\hline 2 & $\begin{array}{l}\text { OD }+1,50 \text { de }(0,5) \\
\text { OE plano }\end{array}$ & Baixa AV OD & Ortotropia; ET' 5 & VB periférica & Observação & $\begin{array}{l}\text { Retratamento após: } \\
\text { AV OD } 0,9 \\
(+0,25-0,50 \times 90) \\
\text { VB periférica }\end{array}$ \\
\hline 3 & $\begin{array}{l}\text { OD plano }(1,0) \\
\text { OE }-0,50 \mathrm{dc} \\
\times 80(1,0)\end{array}$ & $\begin{array}{l}\text { Sensação que } \\
\text { olho entorta }\end{array}$ & sc ET 10; ET' 5 & VB periférica & $\begin{array}{l}\text { Como a refração } \\
\text { estática era } \\
\mathrm{OD}+0,50 \mathrm{de} \\
\mathrm{OE}+0,50 \mathrm{de} \\
-0,50 \mathrm{dc} \times 90 \\
\text { foi prescrito } \\
\text { somente oclusão } \\
\text { 1hora/dia }\end{array}$ & Estável \\
\hline 4 & $\begin{array}{l}\text { OD }+1,25 \text { de } \\
-1,25 \text { dc } \times 180(0,7) \\
\text { OE }+1,00 \text { de } \\
-0,50 \text { dc } \times 175(1,0)\end{array}$ & Astenopia & $\begin{array}{l}\text { sc L ortotropia; } \\
\text { ET' } 4\end{array}$ & VB periférica & Óculos para perto & Estável \\
\hline 5 & $\begin{array}{l}\text { OD plano }(0,9) \\
\text { OE plano }(1,0)\end{array}$ & Sem queixas & sc ET 4; ET' 5 & VB periférica & Observação & $\begin{array}{l}\text { Estável - ET diminuiu } \\
\text { após LASER! }\end{array}$ \\
\hline 6 & $\begin{array}{l}\text { OD }-0,25 \text { de }(1,0) \\
\text { OE }+0,25 \text { de } \\
-0,25 \text { dc } \times 165(1,0)\end{array}$ & $\begin{array}{l}\text { Baixa AV L e P } \\
\text { (AV } 0,4 \text { AO - } \\
\text { com RD }-1,00 \text { de } \\
\text { AO AV }=1,0)\end{array}$ & $\begin{array}{l}\text { sc L ortotropia; } \\
\text { P E } 8\end{array}$ & $\begin{array}{l}\text { VB } \\
\text { Espasmo de } \\
\text { acomodação }\end{array}$ & $\begin{array}{l}\text { Colírio } \\
\text { cicloplégico } \\
\text { por } 1 \text { mês }\end{array}$ & $\begin{array}{l}\text { Óculos para } \\
\text { longe e perto }\end{array}$ \\
\hline 7 & $\begin{array}{l}\text { OD }-0,50 \text { de } \\
-0,50 \mathrm{dc} \times 25(1,0) \\
\text { OE }-0,50 \mathrm{dc} \\
\times 180(1,0)\end{array}$ & Sem queixas & $\begin{array}{l}\text { ET } 12 \text { HTE } 4 ; \\
\text { ET' } 14 \text { HTE } 4\end{array}$ & $\begin{array}{l}\text { Ausência } \\
\text { de VB }\end{array}$ & Óculos para perto & $\begin{array}{l}\text { Com a correção para } \\
\text { perto apresentava } \\
\text { VB periférica, mas } \\
\text { usava raramente e } \\
\text { não possuía queixas }\end{array}$ \\
\hline 8 & $\begin{array}{l}\text { OD }+0,50 \text { de }(0,9) \\
\text { OE plano }(1,0)\end{array}$ & $\begin{array}{l}\text { Sente olho } \\
\text { entortar nas } \\
\text { atividades } \\
\text { para perto }\end{array}$ & $\begin{array}{l}\text { sc } E(T)^{\prime} 15 \\
E(T) 12 \\
\text { cc } E(T)^{\prime} 8 \\
E(T) 8+\text { foria }\end{array}$ & VB periférica & Óculos para perto & $\begin{array}{l}\text { Sua VB piorou } \\
\text { (Titmus 200", Vidros } \\
\text { de Bagolini suprime } \\
\text { para longe). Após } \\
1 \text { mês cc Bagolini } \\
\text { fusão Titmus 200" }\end{array}$ \\
\hline 9 & AO plano $(1,0)$ & Sem queixas & $\begin{array}{l}E^{\prime} 10 \\
\text { E } 4\end{array}$ & VB central & $\begin{array}{l}\text { Oclusão OD } \\
1 \mathrm{~h} / \text { dia }\end{array}$ & Estável \\
\hline 10 & $\begin{array}{l}\text { AO }+1,00 \text { de } \\
-0,50 \text { dc } \times 165\end{array}$ & Sem queixas & $E=E^{\prime} 4$ & VB central & Observação & Estável \\
\hline
\end{tabular}

No caso referido (Quadro 1, paciente 3) foi necessária a correção cirúrgica do estrabismo, pois o controle do desvio não foi obtido mesmo com adaptação de lente de contato no olho míope, com o intuito de corrigir a anisometropia e favorecer a fusão. Possivelmente, à dificuldade de fusão decorrente da monovisão imposta pela sobrecorreção, somou-se instabilidade provocada pela incomitância vertical do desvio. Após a cirurgia o desvio foi totalmente corrigido e paciente não apresentou mais sintomas de astenopia após adaptação de lente de contato no olho míope.
Além da descompensação de desvios pré-existentes, esses estrabismos secundários a monovisão induzida pela cirurgia refrativa também podem ser atribuídos à anisometropia resultante, pois imagens diferentes são projetadas em cada fóvea. Dependendo do grau de anisometropia produzida pela monovisão, pode ocorrer a síndrome da monofixação, que é a perda da fusão bifoveal conseqüente a um escotoma de supressão na fóvea do olho não fixador. O escotoma se transfere de fóvea para fóvea quando a fixação é captada pelo outro olho $^{(11-12)}$. No caso específico da correção por monovisão, a 
qual produz um borramento pela anisometropia em um olho, o olho no qual a fixação ocorre depende da distância do objeto fixado. O olho emétrope, usualmente o dominante, torna-se o fixador para objetos à distância e o olho míope, usualmente o não dominante, torna-se o fixador para perto. Esse padrão de fixação resulta em escotoma alternante na fóvea do olho não fixador ${ }^{(13)}$.

Estudos da visão binocular em um grupo de pacientes adultos com monovisão cirúrgica induzida por longo tempo ${ }^{(13)}$ mostrou que esses pacientes exibem deficiência na visão binocular central, comprovada por redução na estereopsia e na ausência de fusão foveal. Essa deficiência de fusão foveal

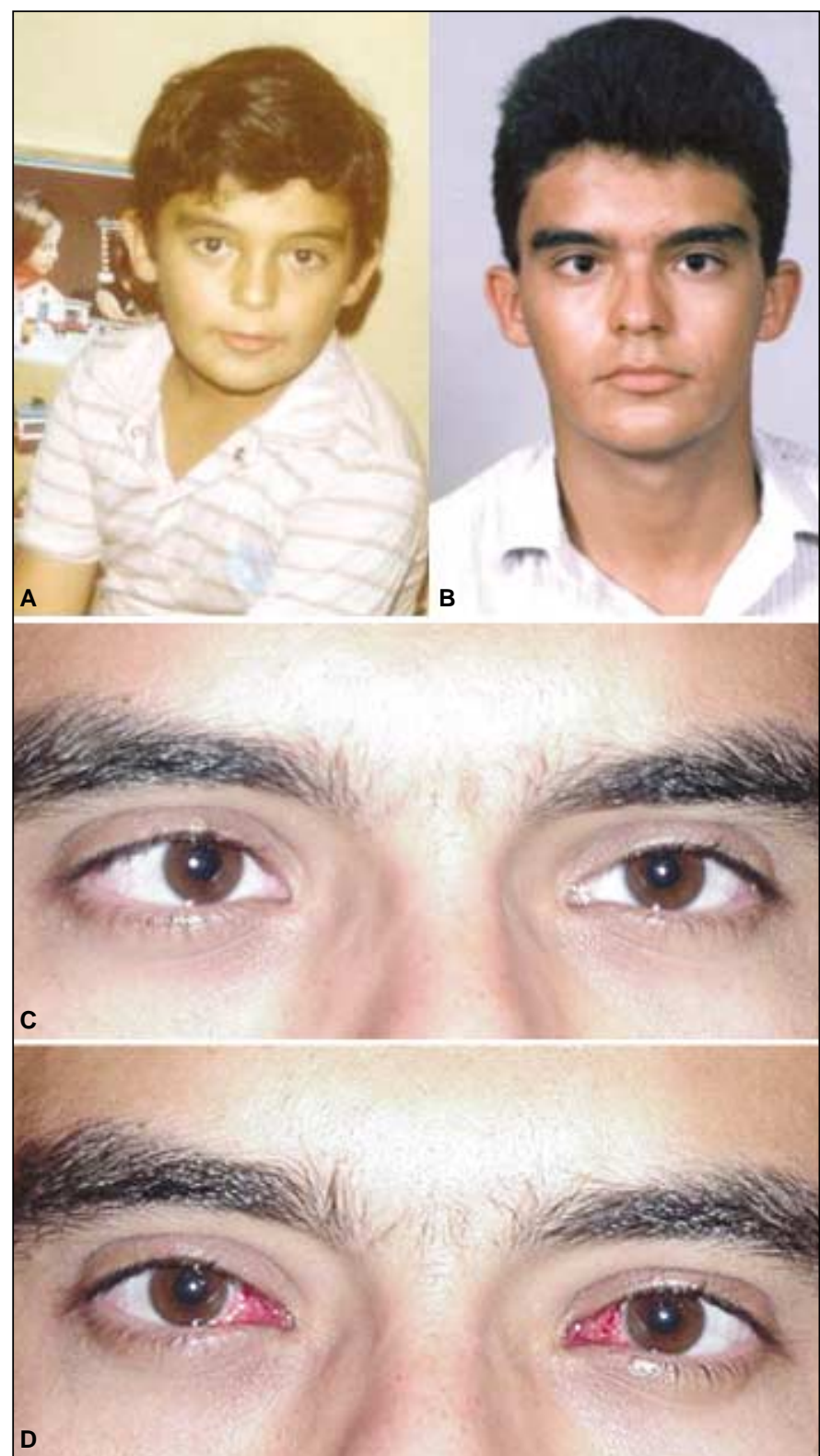

Figura 1 - Fotos do paciente 1 - A: paciente quando criança; B: paciente quando jovem, antes da correção a laser; C: paciente após correção a laser e antes da cirurgia de estrabismo; D: paciente após cirurgia de estrabismo ocorre mesmo se a correção apropriada, para corrigir a anisometropia induzida, foi colocada em uso, ou seja, adultos que possuíam fusão bifoveal podem se tornar monofixadores após uma experiência visual binocular anômala. Os autores concluem que o sistema visual binocular pode ser susceptível a mudanças por toda a vida.

Contrariamente, outros autores ${ }^{(14)}$ relataram haver comprometimento mínimo da visão binocular, avaliada pela acuidade estereoscópica, em pacientes présbitas míopes após monovisão induzida por laser, técnica PRK (ceratectomia fotorrefrativa). Nenhum paciente descrito por esses autores apresentou foria ou desvio ocular, o que pode ser explicado pelo fato de que a monovisão foi efetuada com hipocorreção do olho não dominante em apenas 1,25 dioptrias esféricas.

De modo similar ao que ocorre após monovisão induzida por cirurgia refrativa, estrabismos, caracterizados como heteroforias descompensadas, foram relatados após facecto$\mathrm{mia}^{(15)}$, pois sendo feita a cirurgia em um olho por vez, mesmo usando correção com lentes intra-oculares, pode-se induzir anisometropia, dependendo do estado refrativo do outro olho. A aniseicônia causada pela disparidade de imagens certamente exerce um papel importante no rompimento da fusão, devido à assimetria dos impulsos sensoriais em cada olho. Há também relatos de ruptura da fusão em pacientes com ceratocone assimétrico de longa duração, que mostraram função binocular anômala com microestrabismo e redução da estereoacuidade quando usando lentes de contato ${ }^{(16)}$.

É importante notar que os pacientes do Grupo 1 (pacientes 2 a 5, Quadro 1) possuíam agravantes, ou fatores de descompensação, como a microtropia, ausência de visão binocular central e incomitâncias alfabéticas (Figura 2). Nesses casos é mais esperado haver descompensação do desvio, pois provavelmente esses indivíduos apresentavam fusão periférica instável. A cirurgia fotorrefrativa foi realizada nesses casos em pacientes com estrabismo pré-existente e sem avaliação prévia de suas condições sensoriais.

Sem dúvida, identificar quais são os pacientes que podem desenvolver essas complicações seria o ideal. É o que foi realizado com os pacientes do grupo 2, que tiveram a oportunidade de serem submetidos a exame ortóptico prévio. Nesse exame eram analisadas a amplitude de vergência horizontal, o grau de estereopsia e a presença ou ausência de supressão. Assim, o procedimento a laser só era aprovado quando se constatava visão binocular, mesmo periférica. Os pacientes também eram informados da possibilidade do uso de óculos para detalhes de perto.

Comparando-se os dados dos pacientes do grupo 2 antes e após o laser (Quadros 2 e 3), podemos notar que aqueles que possuíam esotropia acomodativa eram os que apresentavam menos ou nenhuma queixa após o procedimento, e a evolução foi estável ao longo dos meses após a cirurgia. Esses dados sugerem que mesmo na idade adulta, a presença de visão binocular central é fundamental para a estabilidade motora após mudanças repentinas no estado refrativo do olho, como acontece após a correção a laser. Mesmo um paciente (2 - 
Quadro 3) que ficou hipocorrigido em um olho, enquanto aguardava para ser submetido a retratamento, não apresentou sintomas de descompensação de seu estrabismo.

Entretanto, mesmo com os cuidados da avaliação prélaser, alguns pacientes (3,4,5,7,8 - Quadros 2 e 3$)$ sofreram piora em seu estado sensorial. Quando óculos (refração estática, se havia grau residual ou adição, se havia presbiopia) eram prescritos e usados, havia melhora no grau de visão binocular e os sintomas regrediam. Em um paciente (5 - Quadros 2 e 3) houve diminuição do desvio motor, mas não houve alteração no estado sensorial.

Nem todos os pacientes do Grupo 2 apresentavam queixas propriamente de estrabismo. Um dos pacientes do grupo 2 (paciente 6) possuía queixas de embaçamento da visão para longe apesar de ter se tornado praticamente emétrope após o laser. Ao exame, ele apresentava espasmo de acomodação, tratado com colírio cicloplégico por aproximadamente dois meses. Entretanto, ao suspendermos progressivamente a medicação, voltaram os sintomas de borramento visual para longe. Analisando sua história pregressa, esse indivíduo relatava história de tratamento ortóptico na adolescência, como exercícios para insuficiência de convergência e estava hipercorrigido em sua miopia antes do procedimento a laser. Apesar de não haver sinais de alterações na visão binocular, ao exame sensório-motor pré-laser, o treinamento de convergência realizado pelos exercícios e/ou o fato de estar hipercorrigido e a correção fotorrefrativa ter sido baseada na sua refração estática, provavelmente exerceram alguma influência em seu quadro clínico.

\section{CONCLUSÕES}

É muito importante a seleção adequada dos pacientes que desejam correção a laser, principalmente naqueles com história de estrabismo, evitando subestimar alterações que possam comprometer sua capacidade de fusão. Recomendamos que a avaliação pré-operatória para todos os candidatos à cirurgia refrativa seja mais abrangente, e sugerimos:

1) Indagar sempre a história ocular pregressa, incluindo tratamento de ambliopia, estrabismo, anisometropia, uso de prismas ou outros fatores que revelem que os pacientes possam ter baixo controle fusional.

2) Avaliação do estado sensorial e motor:

a) Teste de cobertura simples e alternada, para longe e perto, com e sem correção, incluindo posições de supra
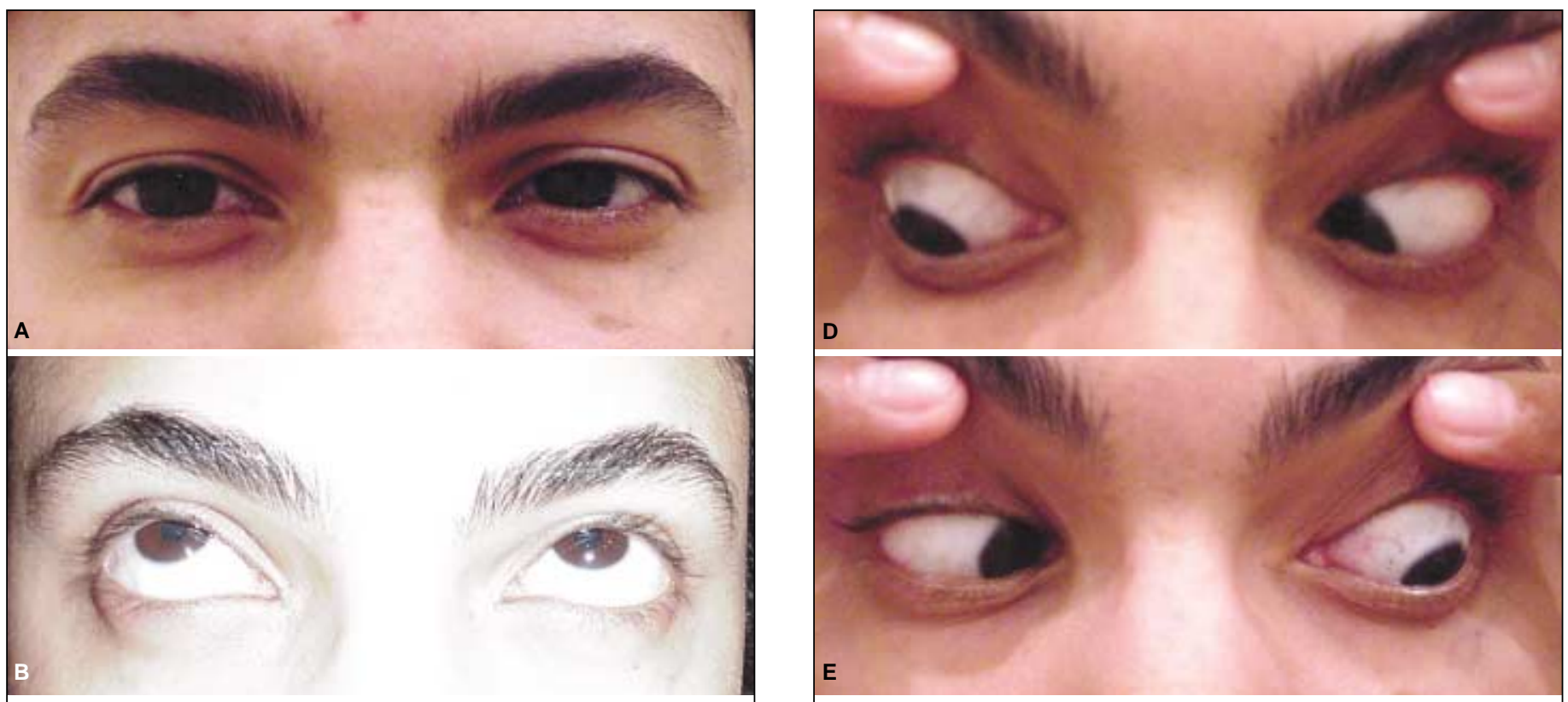

D
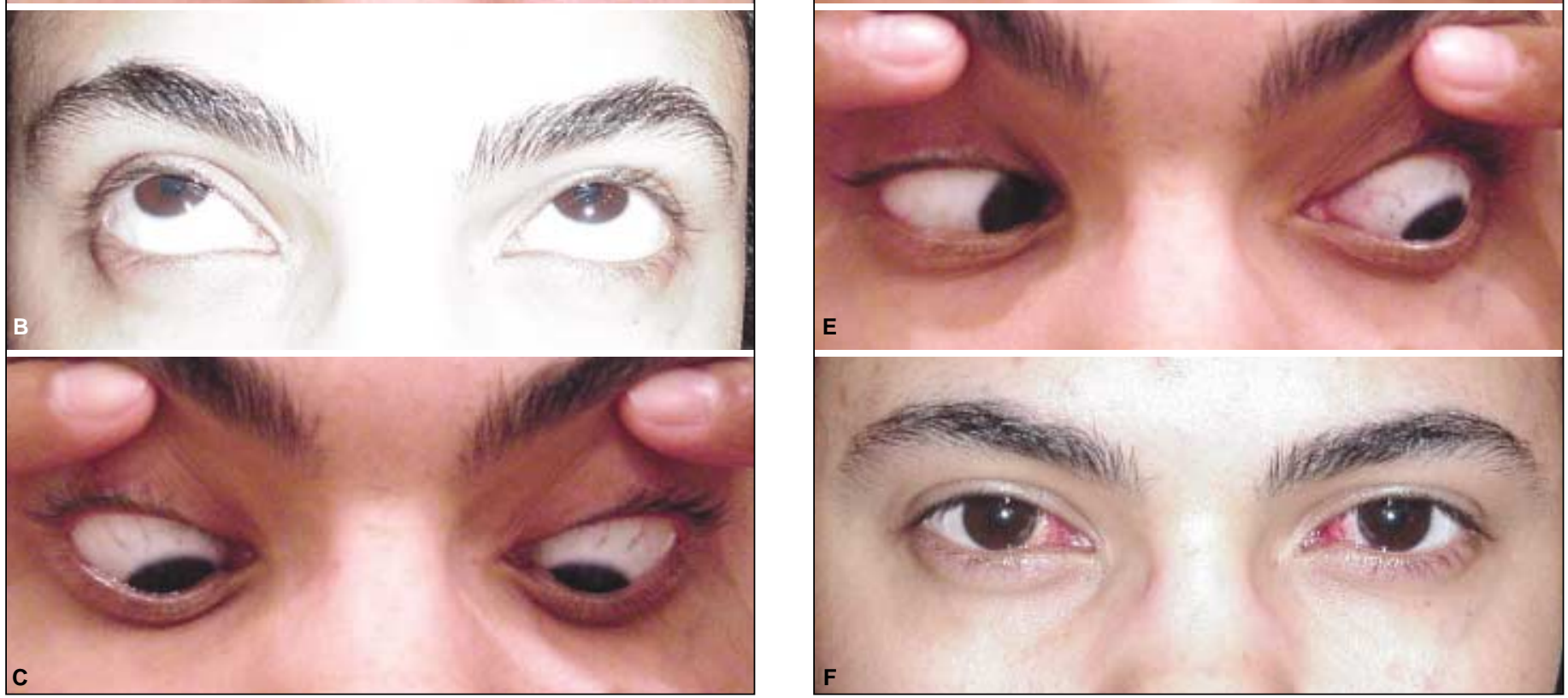

Figura 2 - Fotos do paciente 2 após correção a laser e antes da cirurgia de estrabismo (A: na posição primária do olhar para perto; B: supraversão; C: infraversão; D: infradestroversão; E: infralevoversão) e após cirurgia de estrabismo (F: na posição primária do olhar) 
e infraversão para pesquisar a presença de incomitâncias alfabéticas, que podem ser fatores de descompensação da visão binocular no pós-operatório.

b) Avaliação da visão binocular com o teste de Titmus (avaliação da estereopsia), Luzes de Worth ou Lentes Estriadas de Bagolini (avaliação da supressão).

c) Avaliação do nível de reserva fusional, para seleção dos pacientes de risco a desenvolverem diplopia após o laser.

Além da avaliação pré-operatória mencionada, recomendamos também:

1) Não dificultar a capacidade fusional do paciente, evitando um longo intervalo entre o laser de um olho e outro, o que poderia comprometer a habilidade do paciente fundir as imagens devido à anisometropia ou aniseicônia.

2) Evitar mudar a dominância ocular natural dos pacientes. Se o procedimento for feito em cada olho em tempos diferentes, começar pelo olho dominante - não colocar o olho não dominante em vantagem.

3) Evitar criar monovisão em pacientes com baixo controle fusional, ou se for o caso, fazer um teste de monovisão com lentes de contato e medir novamente a reserva fusional. Se esta for baixa, avisar o paciente de que as chances de perda da binocularidade são grandes.

4) Se forem identificados problemas maiores de estrabismo, aconselhamos a correção do alinhamento ocular antes do laser.

5) Conferir cuidadosamente as lentes dos óculos em uso, pois podem ter prismas ou centros ópticos descentrados em lentes de alto poder refrativo.

6) Redigir termos de consentimento livre, mencionando a possibilidade de complicações relacionadas ao estrabismo.

\section{ABSTRACT}

Purpose: To evaluate patients with strabismus submitted to excimer laser refractive procedure. Methods: Complete eye examination was performed, including motor and sensorial evaluation in 15 patients from October, 2000 to September, 2001. Follow-up was at least 6 months after excimer laser procedure. Patients who had been submitted to laser were named the first group and those who were seen before laser formed the second group. Results: All patients of the first group required strabismus surgery in order to control symptoms of double vision or to correct the ocular deviation. The majority of the patients of the second group had worsening of their sensorial condition and some also presented worsening of their motor conditions. Half of the patients of the second group showed some relief of astenopia when eyeglasses for near were used. Those with accomodative esotropia did not have any or had less symptoms after the laser procedure and the follow-up showed more stable clinical feature. Conclusions: Refractive surgeons must select their patients and avoid underestimating some conditions that might compromise fusion capability after laser. We recommend motor and sensorial evaluation as part of their preoperative work up for candidates for refractive surgery.

Keywords: Laser surgery/adverse effects; Strabismus/surgery; Strabismus/complications

\section{REFERÊNCIAS}

1. Simms PH. Eye market, eye safety behind battle. Wisconsin State J [serial online] 2000 [cited 2004 feb 16]:1A [2 screens]. Avaliable from: URL: http:/ /www.surgicaleyes.org/ubboard/Forum23/html/000652.html

2. Flowers CW, McDonell PJ. Clinical results of excimer laser photorefractive keratectomy for the treatment of myopia. In: Azar DT, editor. Refractive surgery. Stanford: Appleton \& Lange; 1997. p.441-53.

3. Pereira T, Forseto AS, Nosé W. Complicações per e pós-operatórias em 1000 olhos submetidos a LASIK. Arq Bras Oftalmol 2001;64:499-506.

4. Rama P, Chamon W, Genisi C, Azar DT. Excimer laser intrastromal keratomileusis (LASIK). In: Azar DT, editor. Refractive surgery. Stamford: Appleton \& Lange; 199. p.455-69.

5. Marmer RH. Ocular deviation induced by radial keratotomy. Ann Ophthalmol 1987;19:451-2.

6. Zwaan J. Strabismus induced by radial keratotomy. Mil Med 1996;161:630-1.

7. Mandava N, Donnenfeld ED, Owens PL, Kelly SE, Haight DH. Ocular deviation following excimer laser photorefractive keratectomy. J Cataract Refract Surg 1996;22:504-5.

8. Schuler E, Silverberg M, Beade P, Moadel K. Decompensated strabismus after laser in situ keratomileusis. J Cataract Refract Surg 1999;25:1552-3.

9. Kim SK, Lee JB, Han SH, Kim EK. Ocular deviation after unilateral laser in situ keratomileusis. Yonsei Med J 2000;41:404-6.

10. Furr BA, Archer SM, Del Monte MA. Strabismus misadventures in refractive surgery. Am Orthopt J 2001;51:11-5.

11. Parks MM. The monofixation syndrome. Trans Am Ophthalmol Soc 1969;67: 609-57.

12. Weakley DR. The association between anisometropia, amblyopia and binocularity in the absence of strabismus. Trans Am Ophthalmol Soc 1999;97:987-1021.

13. Fawcett SL, Herman WK, Alfieri CD, Castleberry KA, Parks MM, Birch EE. Stereoacuity and foveal fusion in adults with long-standing surgical monovision. J AAPOS 2001;5:342-7.

14. Wright KW, Guemes A, Kapadia MS, Wilson SE. Binocular function and patient satisfaction after monovision induced by myopic photorefractive keratectomy. J Cataract Refract Surg 1999;25:177-82.

15. Hamed LM, Helveston EM, Ellis FD. Persistent binocular diplopia after cataract surgery. Am J Ophthalmol 1987;103:741-4.

16. Sherafat H, White JE, Pullum KW, Adams GG, Sloper JJ. Anomalies of binocular function in patients with longstanding asymmetric keratoconus. $\mathrm{Br}$ J Ophthalmol 2001;85:1057-60.

Nos artigos enviados para publicação, o nome dos autores

e suas afiliações devem estar completos. Isso facilitará a indexação e os links com as bases de dados e o CV Lates. 